\title{
The Relationship between BMI and Age with Lipid Peroxidation, Antioxidant Enzyme Super Oxide Dismutase Glutathione and Serum Homocysteine Level in Polycystic Ovary Syndrome in Sudanese Patients
} Ahmed Nassir Mohamed ${ }^{1}$, Noon Babiker Mohammed ${ }^{2 *}$, Abdelgadir Eltom ${ }^{3}$, Amin Omer Abbas ${ }^{4}$

\author{
${ }^{1}$ Department of Clinical Chemistry, Alfalah Medical Polyclinic, Taif, Saudi Arabia \\ ${ }^{2}$ Associate professor in clinical chemistry University of Science and Technology, Sudan \\ ${ }^{3}$ Medical Laboratories Sciences, College of Health Sciences, Gulf Medical University, Ajman, UAE \\ ${ }^{4}$ Department of Immunohaematology, Albakkari Polyclinic, Almadinah Almunawarah, Saudi Arabia
}

DOI: $10.36348 /$ sijog.2020.v03i11.003

| Received: 03.11.2020 | Accepted: 14.11.2020 | Published: 30.11 .2020

*Corresponding author: Noon Babiker Mohammed

\section{Abstract}

Polycystic ovary syndrome, also known as Stein-Leventhal syndrome is a common health problem that affects teenage girls and young women. PCOS is associated with a wide spectrum of complications in various parts of health, including reproductive, metabolic and psychological features. This Analytical case control study was conducted to Assessment of Lipid peroxidation, antioxidant enzyme Super oxide dismutase, Glutathione and Serum Homocysteine level in Patients with Polycystic Ovary Syndrome in Khartoum State in which include 300 participant divided into 200 case and 100 control, the sample used to measure Homocysteine , Glutathione, Lipid peroxidation and superoxide dismutase level in serum of participant of high body mass index in case group and normal body mass index for the control by using spectrophotometer machine . out of 300 sample divided into 200 case and 100 control, the mean and standard deviation of the total glutathione was found mean 801.30 and the reduced glutathione 132.2 while in control 748.6,103.2 respectively, and for Super oxide dismutase mean and standard deviation was found 225.2, \pm 57.8 in case while in control found $195.5, \pm 25.6$ respectively and Homocysteine mean and standard deviation found $14.9, \pm 2.1$ for case and $12.1, \pm$ 2.5 for control lastly also the mean and standard deviation investigated while the lipid peroxidation was found $3.4, \pm 1.1$ for case and $2.4, \pm 0.7$ Respectively. the total glutathione mean and standard deviation in group of $<5$ years disease duration PCOS found $1559.6, \pm 282.6$ and for duration of $>5$ years of disease the total glutathione mean and standard deviation found $1196.9, \pm 253.9$ regarding superoxide dismutase mean and standard deviation in group of $<5$ years disease duration was found $227.1, \pm 57.4$ and for duration of $>5$ years of disease the superoxide dismutase mean and standard deviation was found $222.4, \pm 58.8$. The Homocysteine mean and standard deviation in group of $<5$ years disease duration was found14.9, \pm 2.2 and for duration of $>5$ years of PCOS disease found 14.8, \pm 1.9 The age interval have low glutathione mean and standard deviation among age of 15-30 year 60(30\%) found 804.5, \pm 118.8 Hemoctoseine mean and standard deviation in age $15-30$ year found $15.3, \pm 2.5$. Polycystic ovarian syndrome could be a dilemma for many women of reproductive ages irrespective of their reproductive disorders; should incorporate assessment of lipid profiles and possibly oxidative stress markers.

Keywords: Polycystic ovary syndrome (PCOS). Lipid peroxidation. antioxidant enzyme Superoxide dismutase. Glutathione, Homocysteine.

Copyright (C) 2020 The Author(s): This is an open-access article distributed under the terms of the Creative Commons Attribution 4.0 International License (CC BY-NC 4.0) which permits unrestricted use, distribution, and reproduction in any medium for non-commercial use provided the original author and source are credited.

\section{INTRODUCTION}

Polycystic ovary syndrome (PCOS) is a major commonly occurring endocrine disorder with prevalence of $5 \%-21 \%$ among reproductive-aged women ${ }^{(1)}$. PCOS is associated with a wide spectrum of complications in various parts of health, including reproductive, metabolic and psychological features. Several mechanisms suggested to contribute to the pathogenesis of PCOS include hormonal imbalance, resistance to insulin and genetic inheritance [1]. The primary cause of PCOS is multifactorial in origin, though hyperandrogenism, polycystic ovaries and 
oligoanovulation are common features in women with PCOS [2].

Hyperandrogenism favors excess luteinizing hormone secretion in fetal life in hypothalamic-pituitary unit programming, bringing about the development of insulin resistance and abdominal obesity [3]. Also, disruption of ovulatory function (anovulation) in PCOS may result from both altered steroid negative feedback regulation of luteinizing hormone and the compensatory hyperinsulinemia caused by insulin resistance [4]. The Rotterdam Consensus Criteria established by the European Society for Human Reproduction and Embryology and American Society for Reproductive Medicine in 2003 dependent on the broad studies during the most recent decades, have been used as diagnosing criteria for PCOS [5]. However, lifestyle modification, meditation, weight loss, herbal medicines have been used in the management of PCOS [1].

PCOS is a heterogeneous disorder that affects at least $7 \%$ of adult women [6] According to the National Institutes of Health Office of Disease Prevention, PCOS affects approximately 5 million women of childbearing age in the U.S. Costs to the U.S. health care system for the identification and management of PCOS are approximately $\$ 4$ billion per year [7].

Research suggests that $5 \%$ to $10 \%$ of females 18 to 44 years of age are affected by PCOS, making it the most common endocrine abnormality among women of reproductive age in the U.S [8]. Women seeking help from health care professionals to resolve issues of obesity, acne, amenorrhea, excessive hair growth, and infertility often receive a diagnosis of PCOS.

\footnotetext{
Women with PCOS have higher rates of endometrial cancer, cardiovascular disease, dyslipidemia, and type-2 diabetes mellitus [9].

\section{General Objective}

To assess the relationship between BMI and Age with Lipid peroxidation, antioxidant enzyme Super oxide dismutase Glutathione and Serum Homocysteine level in Polycystic Ovary Syndrome in Sudanese patients.
}

\section{Specific Objective}

- To correlate relationship between BMI and Lipid peroxidation, antioxidant enzyme Super oxide dismutase Glutathione and Serum Homocysteine level in test group.

- To correlate relationship between age and Lipid peroxidation, antioxidant enzyme Super oxide dismutase Glutathione and Serum Homocysteine level in test group.
MATERIAL AND METHODS

Study Design: Analytical case control.

Study Area: The Study done in Sudan Khartoum State in Adam and Hawa Infertility Center.

Study Period: This study carried during the period between December 2015 up to 2019.

\section{Study Population}

The study conducted on healthy women volunteers (age: 18 - 46 years) patients with clinically proven polycystic ovary syndrome.

Inclusion Criteria: Healthy women volunteers Patients with Poly Cystic Ovary Syndrome.

\section{Control Group}

Normal healthy women volunteers n (100) with normal cycles between $18-46$ years old, acted as a control group.

\section{Study Group}

Volunteers women (age: 18-46 years) patients with clinically proven polycystic ovary syndrome $n$ (200) were chosen for the study as study subjects (patients).

\section{Exclusion Criteria}

Hypertension, diabetic Patients, cancer, ischemic heart disease, renal disease, autoimmune disease, thyroid disease Subjects with history of receiving anti-inflammatory drugs in last 6 months and history or present symptoms of any other stress induced disorder. Hyperprolactinemia, as well as thyroid disease, were excluded.

\section{Sample Size}

100 normal healthy women volunteers with normal cycles between 18 - 46 years old, acted as a control group. And 200 women volunteers (age: $18-46$ years) patients with clinically proven polycystic ovary syndrome were chosen for the study as study subjects (patients).

\section{Ethical Consideration}

- The objective of the study was explained to all individuals participating in this study.

- Informed consent was obtained from the entire participant in the study.

- Health education was provided to all participants.

\section{Data collection and questionnaire}

All the data of this study were obtained from data record. A questionnaire is specific designed to 
obtain information which helps in either including or excluding certain individual in the study.

\section{Sampling Collection}

Blood samples $(7 \mathrm{ml})$ were collected from fasting subjects of the study group after fulfillment of the questionnaire as well as the control group, using disposable syringe and antiseptic alcohol [70\%ethanol] swab for skin. The samples were collected in lithium heparin containers and plain containers. Blood in heparinized containers were gently mixed with the anticoagulant to obtain plasma. And serum obtained after centrifuged plain containers at $10000 \mathrm{rpm}$ for 10 minutes at $-4^{\circ} \mathrm{C}$ using cold centrifuge.

Hemolyzed and lipemic samples were rejected and excluded from the study. The whole blood used immediately after collection for testing Glutathione. Plasma samples were preserved at - " $20{ }^{\circ} \mathrm{C} "$ prior to processing, heparinized plasma for testing Superoxide dismutase while serum sample for testing Homocysteine and Lipid peroxidation (malondialdehyde).

\section{Biochemical measurement and Methodologies \\ Materials Required}

Syringe seals, plain containers, lithium heparin containers, alcohol swab, cotton and marker pens, centrifuge, cleaning solution system.

\section{Measurement of Homocysteine and Principle of the Assay}

Bound or dimerized homocysteine (oxidized form) is reduced to free homocysteine, which then reacts with serine catalyzed by cystathionine betasynthase (CBS) to form cystathionine. Cystathionine in turn is broken down by cystathionine beta-lyase (CBL) Tao form homocysteine, pyruvate and ammonia. Pyruvate is then converted by lactate dehydrogenase (LDH) to lactate with nicotinamide adenine dinucleotide (NADH) as coenzyme. The rate of NADH conversion to NAD+ is directly proportional to the concentration of homocysteine (A340 nm).

\section{Measurement of Glutathione and Principle of the Assay}

Glutathione peroxidase (GPx) is an enzyme found in cytoplasmic and mitochondrial fractions of cells. GPx catalyses the reduction of hydrogen-peroxide and hydroperoxides formed from fatty acids, thus effective lyremoving toxic peroxides from living cells. It plays the important role of protecting cells from potential damage from free radicals formed by peroxide decomposition. Fortress kit is used for the quantitative determination of total Glutathione peroxidase (GPX).GPX catalyses the oxidation of Glutathione (GSH) by cumene hydroperoxide. The oxidised glutathione is converted to the reduced form in the presence of glutathione reductase and NADPH. In this reaction the NADPH is oxidized to NADP+ Simultaneously. The decrease in absorbance at 340nm is then measured.

$$
\begin{aligned}
& \mathrm{ROOH}+2 \mathrm{GSH} \longrightarrow \mathrm{GPXROH}+\mathrm{GSSG}+\mathrm{H} 2 \mathrm{O} \\
& \mathrm{GSSG}+\mathrm{NADPH}+\mathrm{H}+\longrightarrow \mathrm{GRNADP}++2 \mathrm{GSH}
\end{aligned}
$$

\section{Measurement of SUPEROXIDE DISMUTASE and Principle of the Assay}

Test Superoxide dismutase (SOD) role is to accelerate the dismutation of the toxic superoxide radical (02- ), produced during oxidative energy processes, to hydrogen peroxide and molecular oxygen. Fortress method employs xanthine and xanthine oxidase (XOD) to generate superoxide radicals which react with 2-(4-iodophenyl)-3-(4-nitrophenol)-5-

phenyltetrazolium chloride (I.N.T.) to form a red form azandye. The superoxide dismutase activity is then measured by the degree of inhibition of this reaction. One unit of SOD is that which causes a 50\% inhibition of the rate of reduction of INT under the conditions of the assay.

The second step is a $\beta$-elimination reaction which takes place under alkaline conditions. This reaction is mediated by reagent $\mathrm{R} 2(30 \% \mathrm{NaOH})$ which specifically transforms the substitution product (thioether) obtained with GSH into a chromophoricthione which has a maximal absorbance wavelength at $400 \mathrm{~nm}$. This method makes it possible to specifically assay glutathione with only one sampling and one colorimetric measurement. A modification of this method can be used to assay other mercaptans. This is based on the measurement of substitution products, thioethers, which absorb light at $356 \mathrm{~nm}$ in the absence of reagent R2. Because of its simplicity, this method is especially well adapted to the assay of glutathione in large series of biological samples. The main advantage of the method is the specificity for glutathione and it does not require an enzyme as a reagent.

\section{Measurement of Lipid peroxidation (malondialdehyde) and Principle of the Assay}

This assay is based on the reaction of a chromogenic reagent, 2-thiobarbituric acid, with MDA at $25^{\circ} \mathrm{C}$. One molecule of MDA reacts with 2 molecules of 2-thiobarbituric acid via a Knoevenagel-type condensation to yield a chromophore with absorbance maximum at $532 \mathrm{~nm}$.

\section{The body mass index (BMI)}

The body mass index (BMI) or Quetelet index is a value derived from the mass (weight) and height of an individual. The BMI is defined as the body mass divided by the square of the body height and is universally expressed in units of $\mathrm{kg} / \mathrm{m} 2$, resulting from mass in kilograms and height in meters. 
The BMI is an attempt to quantify the amount of tissue mass (muscle, fat, and bone) in an individual, and then categorize that person as underweight, normal weight, overweight, or obese based on that value. However, there is some debate about where on the BMI scale the dividing lines between categories should be placed (10). Commonly accepted BMI ranges are underweight: under 18.5 , normal weight: 18.5 to 25 , overweight: 25 to 30 , obese: over 30 .

\section{Interview and Questionnaire}

The complete clinical and personal history of the subjects will be recorded.

\section{Quality Control}

The precision and accuracy of all methods use in this study were checked each time a batch analyzed by including commercially prepared control sera.

\section{Data Analysis}

The data was analyzed using statistical package of social science (SPSS) computer program using, independent t-test, results was expressed as (mean \pm SD), and significance difference was considered as (P-value <0.05).

\section{RESULTS}

Patient samples were investigated to detect the mean and standard deviation to glutathione, superoxide dismutase, lipid peroxidation and serum Homocysteine, glutathione was identify in two form total and reduced one .out of all 300 sample, were divided into 200 case and 100 control, the mean and standard deviation was found as follow the total glutathione mean 801.30 and the reduced glutathione 132.2 while in control 748.6, 103.2 respectively. The reduced glutathione means \pm standard deviation was found $1174.5, \pm 271.5$ in case and 986.1, \pm 191.5in control. When we look to superoxide dismutase the mean \pm standard deviation was found $225.2, \pm 57.8$ in study group while in control found $195.5, \pm 25.6$ respectively. Homocysteine mean \pm standard deviation found $14.9, \pm 2.1$ for case and 12.1, \pm 2.5 for control lastly also the mean and standard deviation investigated for lipid peroxidation and found $3.4, \pm 1.1$ for case and $2.4, \pm 0.7$ Respectively, In this study we found that the level of all parameters in study group was significantly increase when compared to healthy individuals (P.value 0.00) Table-1.

Table-1: Result of tests with Study Subjects and controls group

\begin{tabular}{|l|l|l|l|l|}
\hline Parameters & Mean & Std. Deviation & \multirow{2}{*}{ p.value } \\
\hline \multirow{2}{*}{ Reduce glutathione } & Study Subjects & 801.3 & 132.2 & 0.00 \\
\cline { 2 - 4 } & Controls & 748.6 & 103.2 & \\
\hline \multirow{2}{*}{ Total glutathione } & Study Subjects & 1174.5 & 271.5 & \multirow{2}{*}{0.00} \\
\cline { 2 - 4 } & Controls & 986.1 & 191.5 & \\
\hline superoxide dismutase & Study Subjects & 225.2 & 57.8 & \multirow{2}{*}{0.00} \\
\cline { 2 - 4 } & Controls & 195.5 & 25.6 & \multirow{2}{*}{0.00} \\
\hline \multirow{2}{*}{ Homocysteine } & Study Subjects & 14.9 & 2.1 & \\
\cline { 2 - 4 } & Controls & 12.1 & 2.5 & \\
\hline lipid peroxidation & Study Subjects & 3.4 & 1.1 & \\
\cline { 2 - 4 } & Controls & 2.4 & 0.7 & \\
\hline
\end{tabular}

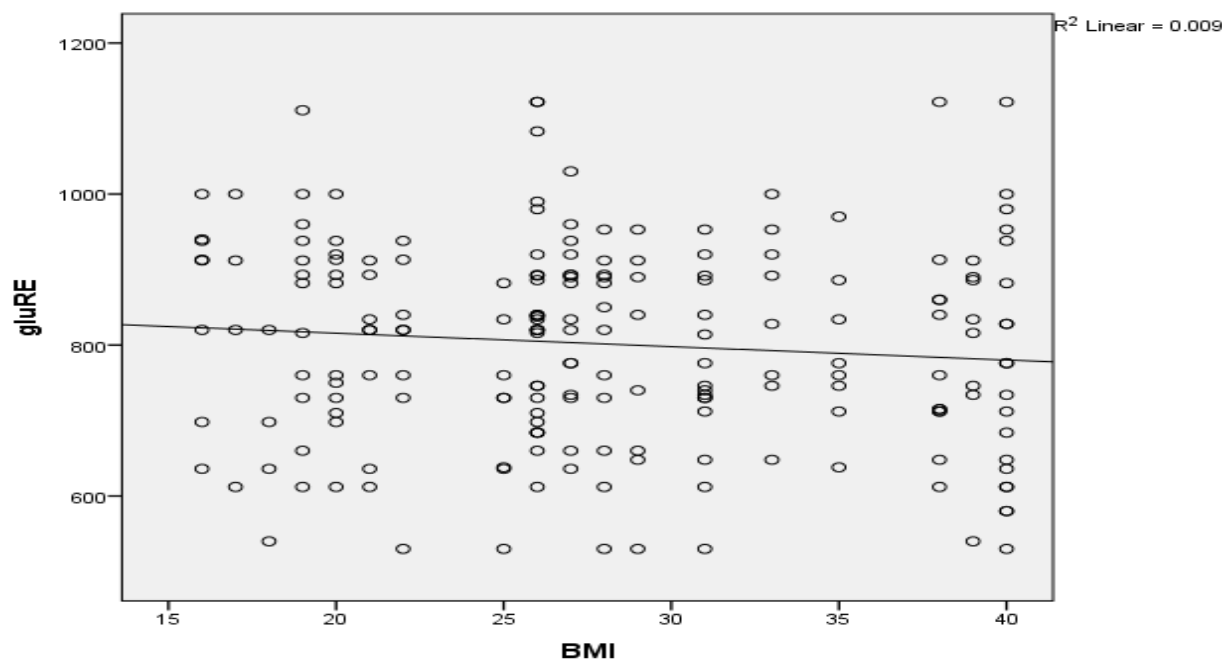

Fig-1: Positive Correlation between Reduced glutathione and BMI (p.value:0.3 r: 0.009) 


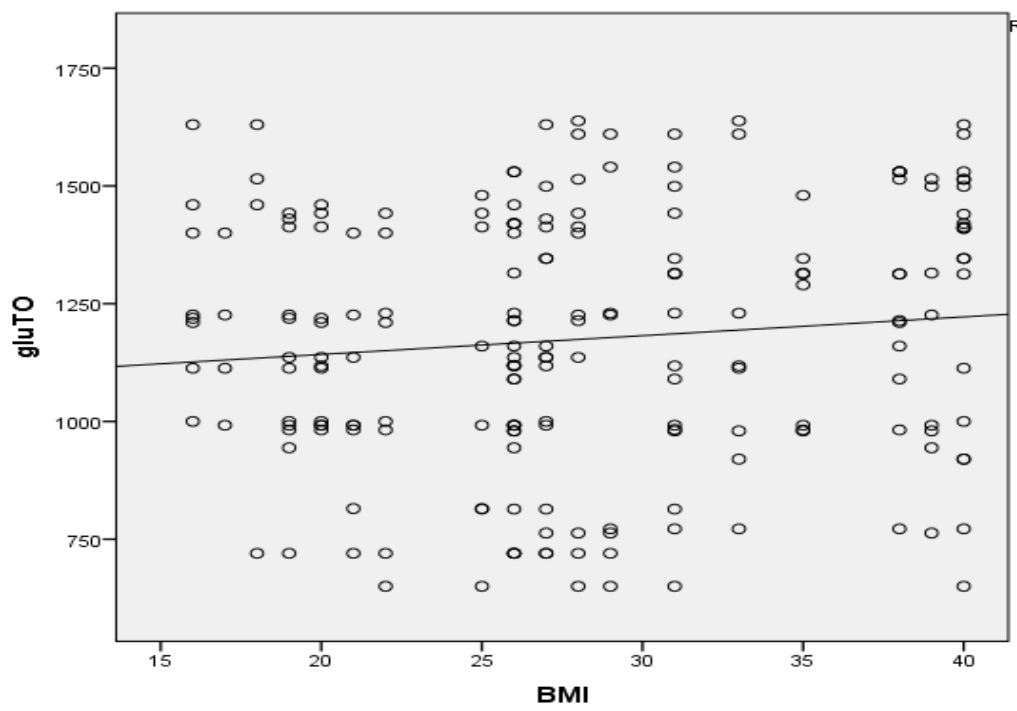

$R^{2}$ Linear $=0.011$

Fig-2: Positive Correlation between Total glutathione and BMI (p.value:0.9 r: 0.011)

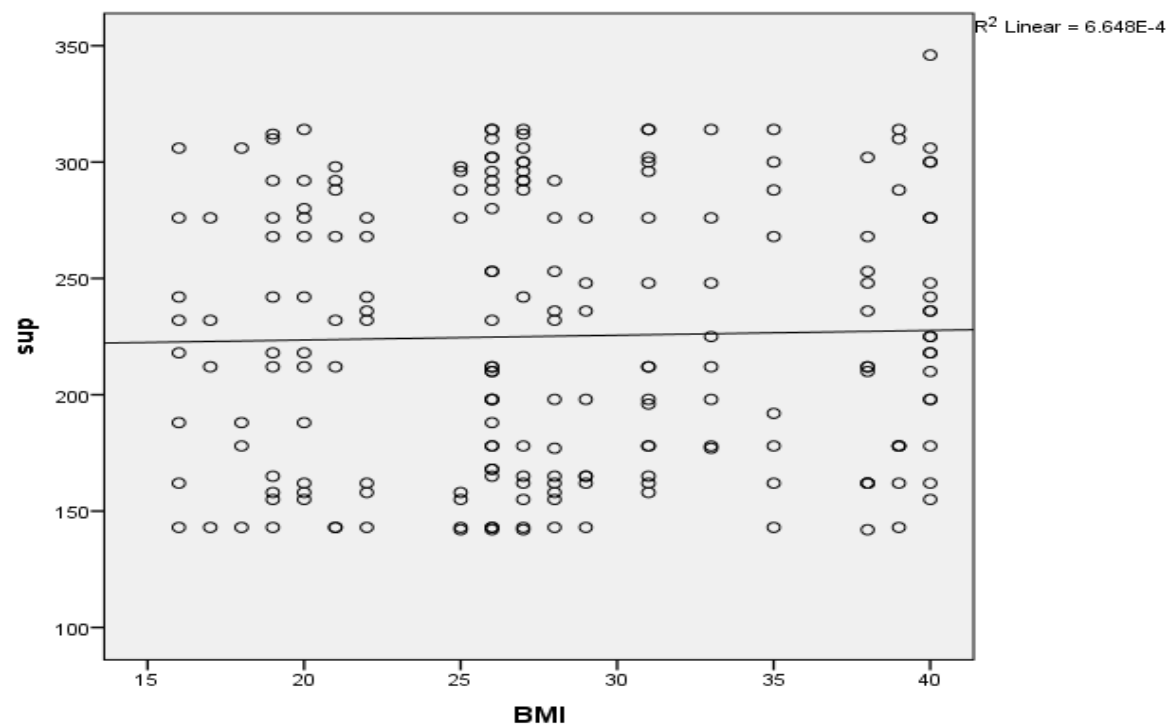

Fig-3: Positive Correlation between superoxide dismutase and BMI (p.value:0.6 r:6.64)

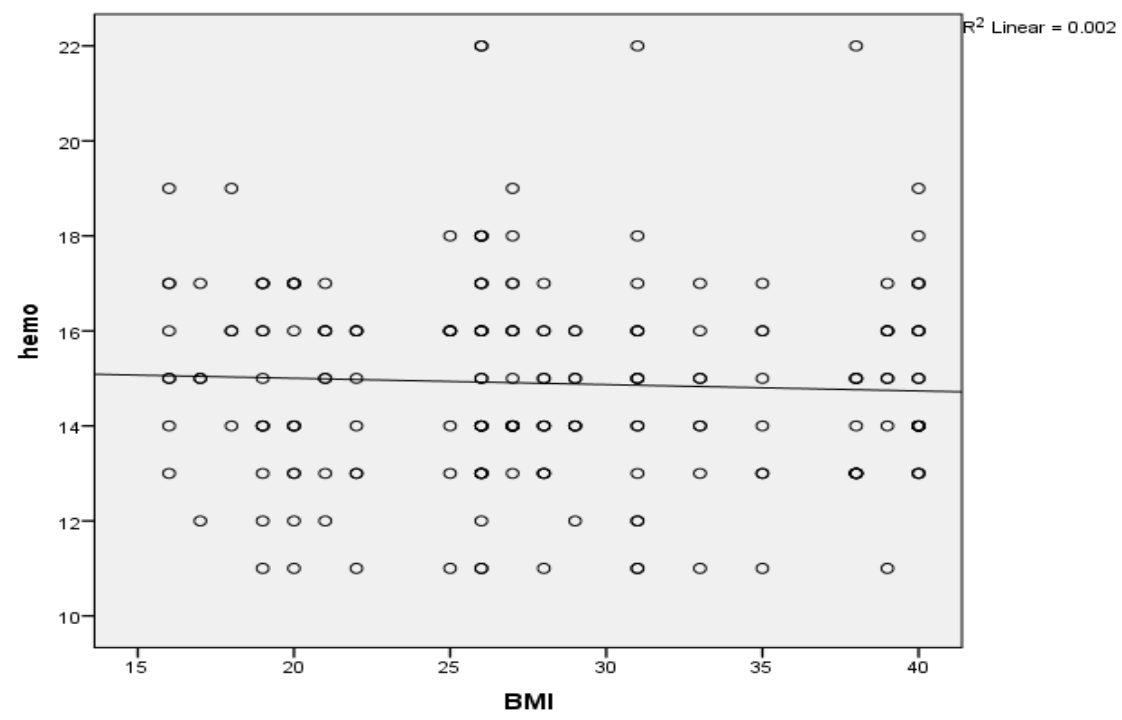

Fig-4: Positive Correlation between Homocysteine with BMI (p.value:0.8 r: 0.002) 


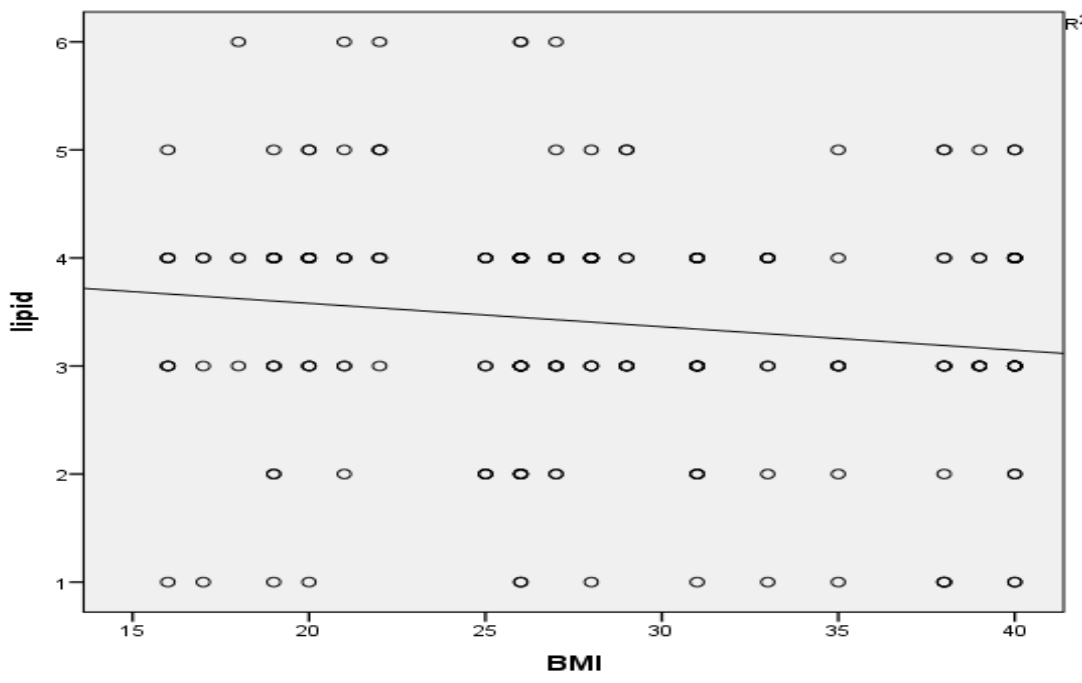

Fig-5: Positive Correlation between lipid peroxidation and BMI (p.value:0.02 r: 0.020)

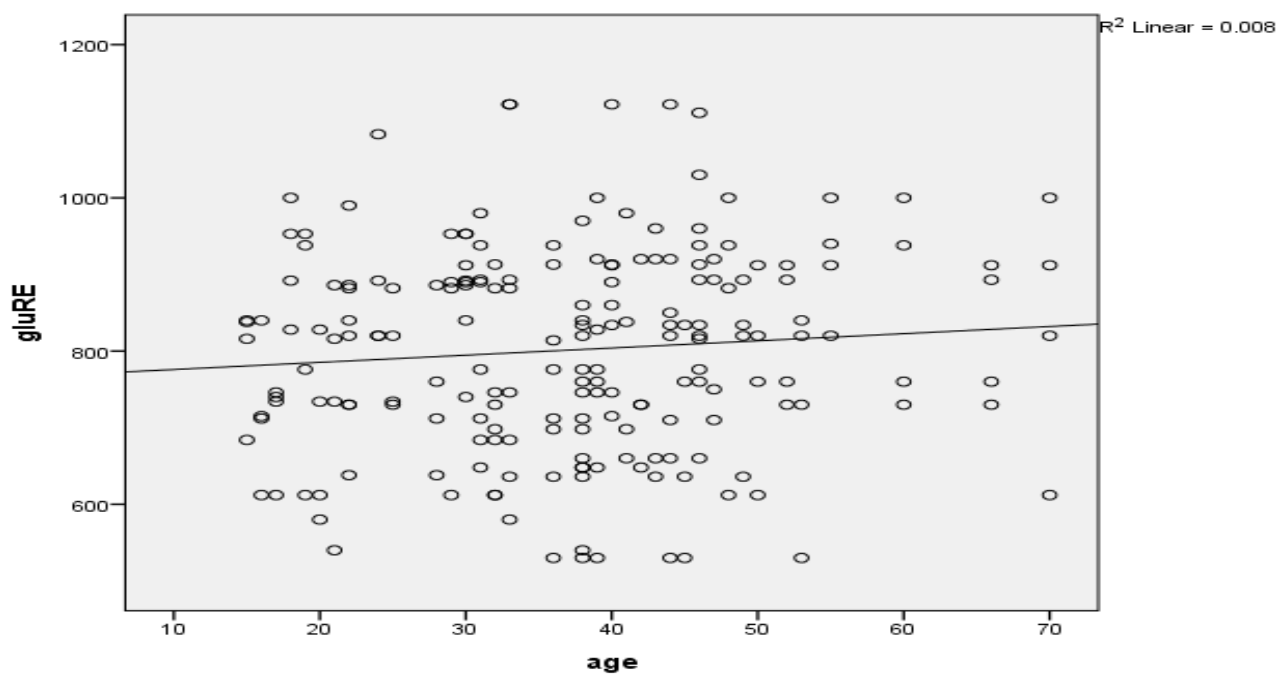

Fig-6: Positive Correlation between Reduced glutathione and age (p.value:0.2 r: 0.008)

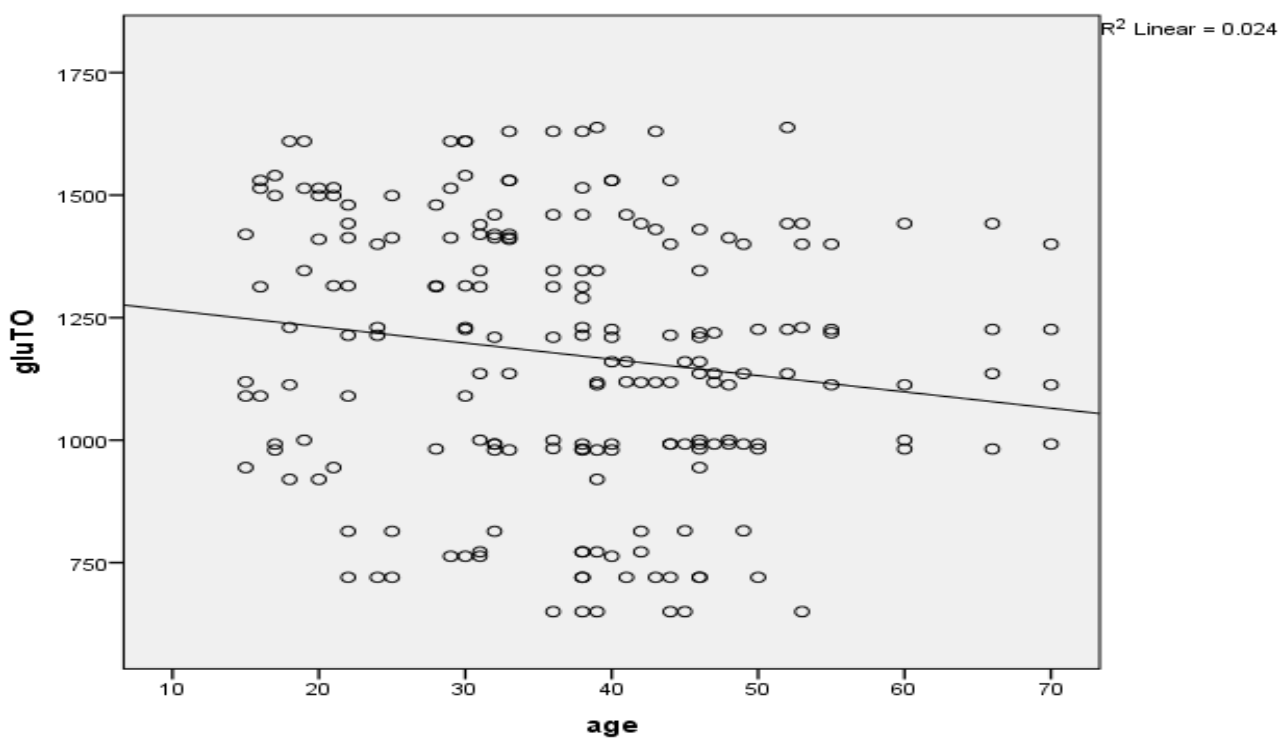

Fig-7: Positive Correlation between Total glutathione and age (p.value:0.01 r: 0.024) 


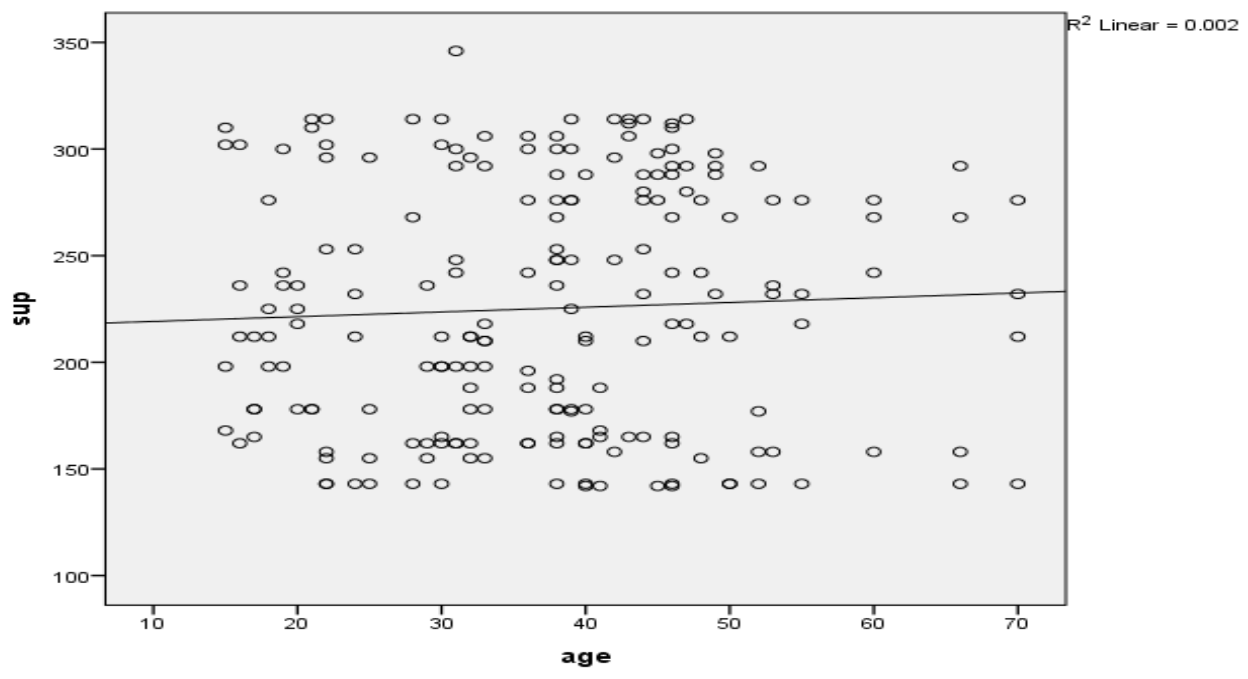

Fig-8: Positive Correlation between superoxide dismutase and age (p.value:0.3 r: 0.002)

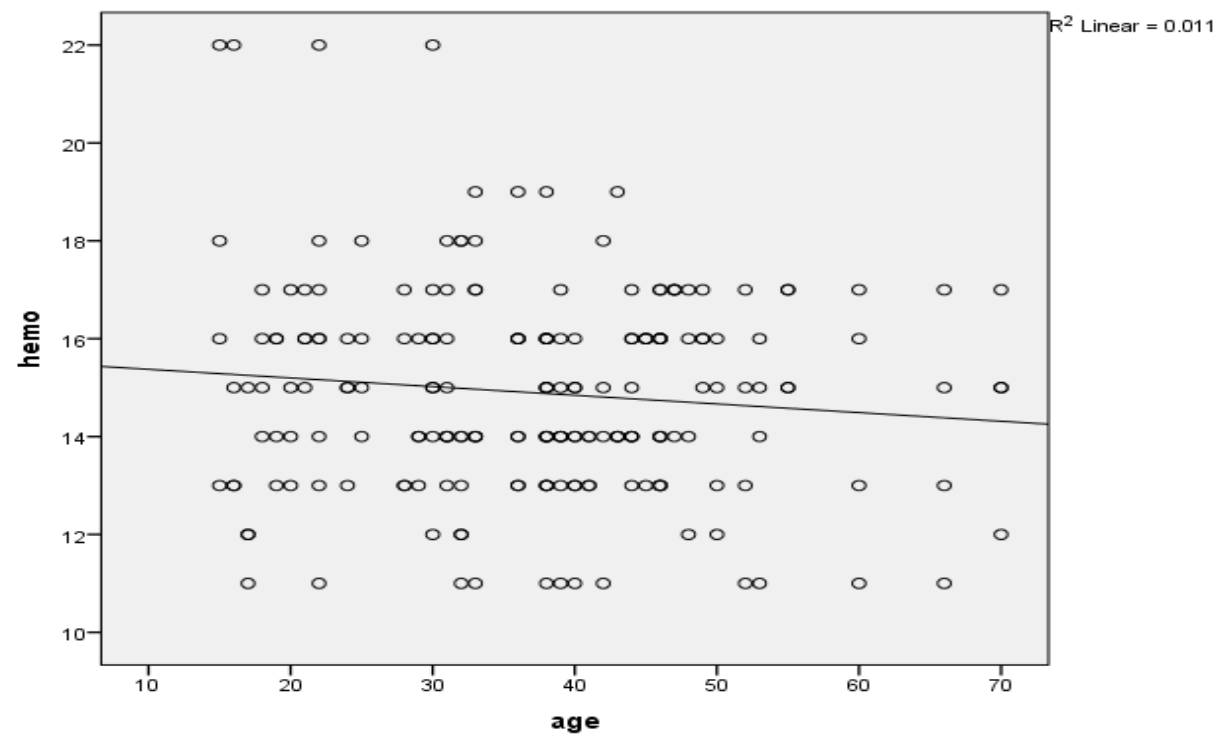

Fig-9: Positive Correlation between Homocysteine and age (p.value:0.1 r: 0.011)

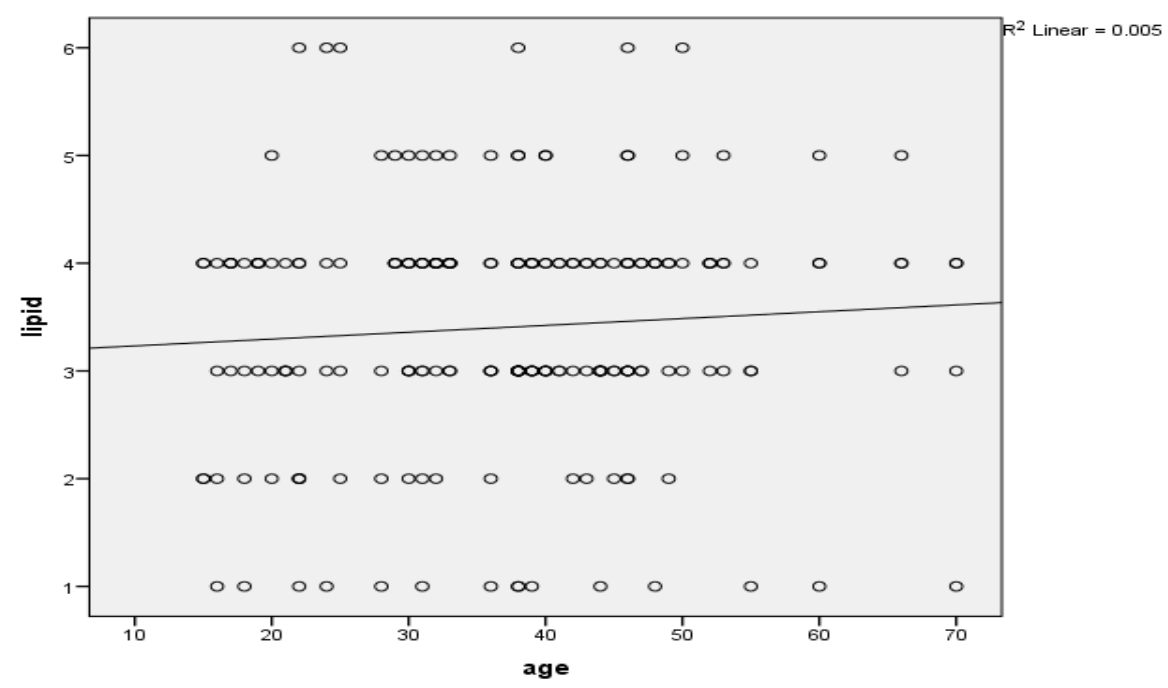

Fig-10: Positive Correlation between lipid peroxidation and age (p.value:0.4 r: 0.005) 


\section{DISCUSSION}

Polycystic ovarian syndrome (PCOS) is one of the most common endocrine abnormalities among women in reproductive ages.

In the present study the lipid peroxidation product levels have been increased significantly in the patients with polycystic ovary syndrome compared to controls. In our study the antioxidant enzymes i.e. SOD \& GPx activities have been increased significantly in patients with PCOS compared to controls. SOD is the important antioxidant enzyme having an antitoxic effect against super oxide anion. The over expression of SOD might be an adaptive response and it results in increased dismutation of superoxide to hydrogen peroxide. GPX, an oxidative stress inducible enzyme plays a significant role in the peroxyl scavenging mechanism and in maintaining functional integration of the cell membranes [10]. The rise could be due to its induction to counter the effect of increased oxidative stress.

After adjusting for confounding factors such as age, BMI, gravidity, and serum lipid levels [11] increased lipid peroxidation have been reported in the endometrium of women with PCOS [12, 13]. However, several studies failed to find significant differences in the, lipid peroxide, and PCOS in women

The Glutathione is a group of multifunctional proteins, which play a central role in detoxification of electrophilic chemicals \& the hepatic removal of potentially harmful hydrophobic compounds from blood [14]. We have observed a significant increase in the GS activity in patients with polycystic ovary syndrome compared to controls in which out of 300 sample divided into 200 study group and 100 control group, the mean and standard deviation was found as follow the total glutathione mean 801.30 and the reduced glutathione 132.2 while in control 748.6,103.2 respectively. The rise in the activity of GS could be due to its induction to counter the effect against increased oxidative stress.

Homocysteine has been recognized recently as a risk factor for vascular diseases. In our study, Serum. Our data showed that serum Homocysteine levels were significantly higher in PCOS women than controls. Our findings are consistent with a previous study by Loverro and his group [15] and Badawy et al., [16]. Mancini et al., [17] in their study found no significant difference in Homocysteine levels among PCOS women and controls.

Difference Studies have shown correlation between Homocysteine and BMI [18]. Schachter [19], shown correlation between Homocysteine and insulin resistance. Studies found that increased Homocysteine levels and decreased antioxidant capacity may contribute to the increased risk of cardiovascular disease in women with PCOS [20]. Other studies have evaluated Homocysteine levels in PCOS subjects based on BMI matched subjects. In our study Homocysteine levels were compared among PCOS cases and controls as well as in subgroups based on BMI and waist circumference [21]. An increased serum Homocysteine level is associated with the formation of atherosclerotic plaques and myocardial infarction. Similar reports of increased levels of Homocysteine in polycystic ovary syndrome were reported [22]. With multiple regression analysis the reduction in antioxidants along with significant increase in the lipid peroxidation, antioxidant enzymes and Homocysteine levels was calculated to be independent from the BMI in patients with Poly Cystic Ovary Syndrome (PCOS) compared to Controls $(p>0.08)$ and these parameters were considered as the independent determinants insignificantly increased oxidative stress and increased Homocysteine levels in patients with PCOS.

\section{CONCLUSIONS}

- The present study has demonstrated that mean serum Homocysteine, glutathione, serum lipid peroxidase superoxide concentrations were increased in women with PCOS. The increase was more pronounced with increase in BMI and age. Higher levels were observed in obese cases when compared with control cases.

- We have observed a significant increase in the GS activity in patients with polycystic ovary syndrome compared to controls.

- Regression analysis the reduction in antioxidants along with significant increase in the lipid peroxidation, antioxidant enzymes and Homocysteine levels was calculated to be independent from the BMI.

\section{RECOMMENDATIONS}

1. Polycystic ovarian syndrome could be a dilemma for many women of reproductive ages with many unfavorable outcomes mentioned to evaluation of PCOS patients

2. irrespective of their reproductive disorders; should incorporate assessment of lipid profiles and possibly oxidative stress markers

3. Interpretation of the results of these analytes may necessitate possible referral to cardiologists for further assessment of cardiovascular disease risks in these patients.

4. Furthermore, the assessment of obesity in PCOS patients should routinely incorporate $\mathrm{WC}$ and $\mathrm{WC}$ : $\mathrm{HC}$ ratio indices, addition to BMI.

5. Education of population about the relation between antioxidant and BMI.

\section{REFERENCES}

1. Amini, L., Tehranian, N., Movahedin, M., Tehrani, F. R., \& Ziaee, S. (2015). Antioxidants and management of polycystic ovary syndrome in 
Iran: A systematic review of clinical trials. Iranian journal of reproductive medicine, 13(1), 1-8.

2. Norman, R. J., Dewailly, D., Legro, R. S., \& Hickey, T. E. (2007). Polycystic ovary syndrome. The Lancet, 370(9588), 685-697.

3. Franks, S., \& Berga, S. L. (2012). Does PCOS have developmental origins?. Fertility and sterility, 97(1), 2-6.

4. Abbott, D. H., Barnett, D. K., Bruns, C. M., \& Dumesic, D. A. (2005). Androgen excess fetal programming of female reproduction: a developmental aetiology for polycystic ovary syndrome?. Human reproduction update, 11(4), 357-374.

5. Joham, A. E., Teede, H. J., Ranasinha, S., Zoungas, S., \& Boyle, J. (2015). Prevalence of infertility and use of fertility treatment in women with polycystic ovary syndrome: data from a large community-based cohort study. Journal of women's health, 24(4), 299-307.

6. Aubuchon, M., \& Legro, R. S. (2011). Polycystic ovary syndrome: current infertility management. Clinical obstetrics and gynecology, 54(4), 675-684.

7. American Congress of Obstetricians and Gynecologists. (2009). ACOG Practice Bulletin No. 108: Polycystic Ovary Syndrome. Obstet Gynecol. 114(4):936-949.

8. National Institutes of Health. (2008). Department of Health and Human Services. Beyond Infertility: Polycystic Ovary Syndrome (PCOS). NIH Pub. No. 08-5863.

9. McFarland, C. (2012). Treating polycystic ovary syndrome and infertility. $M C N$ : The American Journal of Maternal/Child Nursing, 37(2), 116121.

10. Chandra, R., Aneja, R., Rewal, C., Konduri, R., Dass, S. K., \& Agarwal, S. (2000). An opium alkaloid-Papaverine ameliorates ethanol-induced hepatotoxicity: diminution of oxidative stress. Indian Journal of Clinical Biochemistry, 15(2), 155-160.

11. Jenkins, C., Wilson, R., Roberts, J., Miller, H., McKillop, J. H., \& Walker, J. J. (2000). Antioxidants: their role in pregnancy and miscarriage. Antioxidants and Redox Signaling, 2(3), 623-628.

12. Szczepańska, M., Koźlik, J., Skrzypczak, J., \& Mikołajczyk, M. (2003). Oxidative stress may be a piece in the endometriosis puzzle. Fertility and sterility, 79(6), 1288-1293.

13. Gupta, S., Agarwal, A., Krajcir, N., \& Alvarez, J. G. (2006). Role of oxidative stress in endometriosis. Reproductive

biomedicine online, 13(1), 126-134.

14. Smith, G. J., Ohl, V. S., \& Litwack, G. (1977). Ligandin, the glutathione S-transferases, and chemically induced hepatocarcinogenesis: a review. Cancer Research, 37(1), 8-14.

15. Loverro, G., Lorusso, F., Mei, L., Depalo, R., Cormio, G., \& Selvaggi, L. (2002). The plasma homocysteine levels are increased in polycystic ovary syndrome. Gynecologic and obstetric investigation, 53(3), 157-162.

16. Badawy, A., State, O., Abd El Gawad, S. S., \& Abd El Aziz, O. (2007). Plasma homocysteine and polycystic ovary syndrome: the missed link. European Journal of Obstetrics \& Gynecology and Reproductive Biology, 131(1), 68-72.

17. Mancini, F., Cianciosi, A., Reggiani, G. M., Facchinetti, F., Battaglia, C., \& de Aloysio, D. (2009). Endothelial function and its relationship to leptin, homocysteine, and insulin resistance in lean and overweight eumenorrheic women and PCOS patients: a pilot study. Fertility and sterility, 91(6), 2537-2544.

18. Tarkun, İ., Çetinarslan, B., Cantürk, Z., \& Türemen, E. (2005). The Plasma homocysteine concentrations and relationship with insulin resistance in young women with polycystic ovary syndrome. Turkish journal of Endocrinology and Metabolism, 1, 23-28.

19. Mohan, S. K., \& Priya, V. V. (2009). Lipid peroxidation, glutathione, ascorbic acid, vitamin $\mathrm{E}$, antioxidant enzyme and serum homocysteine status in patients with polycystic ovary syndrome. Biology and Medicine, 1(3), 44-49.

20. Salehpour, S. (2011). Evaluation of homocysteine levels in patients with polycystic ovarian syndrome. International journal of fertility \& sterility, 4(4), 168-171.

21. Schachter, M., Raziel, A., Friedler, S., Strassburger, D., Bern, O., \& Ron- El, R. (2003). Insulin resistance in patients with polycystic ovary syndrome is associated with elevated plasma homocysteine. Human reproduction, 18(4), 721 727.

22. Badawy, A., State, O., Abd El Gawad, S. S., \& Abd El Aziz, O. (2007). Plasma homocysteine and polycystic ovary syndrome: the missed link. European Journal of Obstetrics \& Gynecology and Reproductive Biology, 131(1), 68-72. 